\title{
A Novel Methodology for Usability Assesment of Rheological Soft Tissue Models
}

\author{
Árpád Takács*§, Péter Galambos*, Imre J. Rudas*, Tamás Haidegger*§ \\ *Antal Bejczy Center for Intelligent Robotics, Óbuda University, Bécsi út 96/b, 1034 Budapest, Hungary

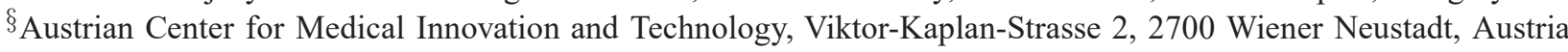 \\ E-mail: \{arpad.takacs, peter.galambos,imre.rudas, tamas.haidegger\}@irob.uni-obuda.hu
}

\begin{abstract}
The task to distinguish between soft tissues by testing their mechanical properties is often referred to as the primary cognitive role of haptic devices. It is a common view that today's surgical simulators that are using haptic interfaces should rely on simple mechanical models of soft tissues, instead of complex, parameterized finite element models, thus enhancing real-time operation and focusing on the most representative mechanical effects. This paper proposes a user-trial validation method for tissue models and their polytopic representation by creating an experimental framework for soft tissue characterization, using the da Vinci Research Kit. The characterization methdodology relies on haptic feedback from the manipulated real tissue, extending the functionality of surgical simulators using virtual tissue models created by the previously proposed soft tissue modeling method. Results showed that the tissue model represents the tissue behavior sufficiently well for use in haptic simulators, based on user experience. Furthermore, it was concluded that silicon phantoms can mimic the behavior of real tissues in various surgical scenarios, especially when using teleoperation manipulation.
\end{abstract}

\section{INTRODUCTION}

In recent years, the number of minimally invasive surgical procedures (MIS) has increased significantly. MIS allows shorter patient recovery time and the decrease of surgical trauma. However, due to the long, rigid design of the MIS tools, the limited vision and confined operation space, several ergonomic difficulties and limitations have arose, that are yet to be solved. These include the deprivation of dexterity, loss of depth perception due to the two-dimensional video image feedback, distributed hand-eye coordination and special tool manipulation, and most importantly, the loss of tactile feedback [1]. While most of these limitations were addressed and partially solved with the introduction of robot-assisted MIS and telesurgery, by using stereo visual feedback, tremor filtering and ergonomic human-machine interfaces (HMIs), the lack of force feedback limits the ability of the surgeon during organ palpation, tumor localization and the identification of other anatomical structures during surgery [2].

The role of haptic feedback in telesurgery is twofold: on one hand, restoring tactile information is essential for assessing the surface properties of the investigated organs. This feature is generally useful for artery and lump detection, therefore the lack of tactile feedback leads to a more difficult localization of palpable anomalies, such as kidney stones. On the other hand, haptics may provide a realistic force feedback to the robot operator (the surgeon), providing information about the mechanical characteristics of the tissue. Haptic feedback improves the quality of basic surgical maneuvers (grabbing, palpation, cutting), allows collision detection and the application of virtual fixtures both in the case of intra-operative scenarios and surgical simulators [3]. Tissue characterization also requires complex perception of the operating environment, where beside tissue stiffness (hardness), relaxation properties and other viscoelastic phenomena can also be investigated and accounted for, when using haptic feedback. It was also shown that for tissue characterization tasks, utilizing force feedback leads to better results than only visual feedback, while, with the combination of the two, superior results can be achieved [4].

There is no general consensus among laparoscopic surgeons, if, and at what level would haptic feedback improve the outcome of procedures. According to many surgeons, having visual feedback alone provides adequate information about the tissue palpation force for safe and reliable operation, however, the lack of haptic feedback is often considered as a major limitation in robot-assisted MIS procedures [5]. Clearly, an experienced surgeon finds the lack of haptic feedback less disturbing, than a novice. However, in haptic guidance, learning spatiotemporal trajectories, contrary motion compensation and strategy planning, the presence of haptic feedback and/or surgical simulators can enhance force skill learning for trainees [6]. In recent years, the use of surgical simulators have largely increased, offering different training scenarios, anatomical variations and conditions in the operating environment [7].

The problem of distinguishing between soft tissues by testing their mechanical properties is often referred to as the cognitive role of haptic devices in simulation environments [8]. By using bilateral haptic devices and accounting for the tissue dynamics, one can solve issues arising from communication latency and high computational requirements [9]. Stability and accuracy deterioration caused by latency and other external disturbances, such as contacting hard tissues or elastic tool deformation, can also be addressed using realistic soft tissue models, their integration into model based force control algorithms largely increase the robustness and reliability of robotassisted interventions [10].

This paper presents a novel methodology for testing the 
usability of soft tissue models in robot-assisted MIS setups, focusing on the modeled mechanical properties of soft tissues and their integration into surgical simulators with haptic capabilities.

\section{RELATED WORK}

The integration of soft tissue properties to robot-assisted and virtual reality based MIS procedures is an actively researched topic within the field of surgical robotics. Methods for acquiring useful measurement data use a combined experimental procedure of measuring tissue relaxation force under step-like tissue compression and force measurement during constant compression rate indentation input. Samur et al. proposed a method for tissue parameter estimation using a custom indenter during laparoscopic surgery, using inverse finite element solution to estimate optimum values of nonlinear hyperelastic and elastic properties [11]. Beccani et al. developed a tool for intra-operative wireless tissue palpation, using a cylindrical palpation probe, estimating local volumetric stiffness values, assuming linear elastic behavior of the tissue [12].

A deformable model based on nonlinear elasticity and finite element method for haptic surgical simulators was proposed in [13], validated on real-time simulations of laparoscopic surgical gestures on virtual liver models. Trejos et al. suggested an augmented hybrid impedance control scheme to perform force control, providing model-based control background for tactile sensing instrument in intra-operative tissue palpation [14]. Endoscopically guided, minimally invasive cannulation tasks were investigated by Wagner et al., testing the hypothesis that force feedback can improve surgical performance, finding that applied forces by the surgeons can be decreased for those with adequate training background [15]. In [1], the authors developed an automated laparoscopic grasper with force feedback capability, in order to aid the surgeons in differentiating tissue stiffness through the PHANToM (Sensable Technologies, Woburn, MA) haptic device. Participants were asked to differentiate between tissues, having provided visual and/or haptic feedback to complete the task.

The viscoelastic tissue model used in this paper is taken from our previous work on soft tissue modeling [16], implemented as a parameter-dependent, discretized virtual model based on the Tensor Product model transformation, as derived in [17]. The aim of this research is to provide a general methodology for addressing the usability and validity range of the proposed tool-tissue interaction model in telesurgical scenarios, where haptic feedback is available.

\section{A Methodology for Model Evaluation AND USABILITY}

Tissue samples with different mechanical properties were investigated during the experiments, which were completed in two phases: Phase I and Phase II. During Phase I, the artificial tissues samples were selected from a wide range of stiffness, being compared to two very different ex-vivo soft tissue samples: chicken liver and chicken breast. After the evaluation of Phase I, new artificial tissues samples were created, aiming to match the mechanical properties of the selected ex-vivo tissue sample. In Phase I, 2 ex-vivo samples and 3 artificial tissue phantoms were prepared for this task. In Phase II, a refined set of 14 artificial tissue phantoms were compared to a single ex-vivo chicken breast sample, as explained in Section III-A. Experiments were carried out using the da Vinci Research Kit, a collection of retired firstgeneration da Vinci Surgical System components, integrated in the Computer Integrated Surgical Systems and Technology (CISST) toolkit [18]. Force sensing was achieved using an OptoForce (OptoForce Ltd., Budapest, Hungary) 3 Degree-ofFreedom (DoF) force sensor. The instruments were integrated using the Robot Operating System (ROS) Indigo version under a 64 bit Ubuntu 14.04 LTS operating system. The ROS packages were based on the $08 / 2016$ release of the Johns Hopkins University sawIntuitiveResearchKit distribution ${ }^{1}$.

\section{A. Experimental Methodology}

In both phases of the experiments, the first step was to determine the mechanical properties of the samples, using curve fitting on the nonlinear mass-spring-damper Wiechert model, following the experimental setup published in [16]. After the acquisition of the parameters, participants were asked to carry out remote $1 \mathrm{DoF}$, axial palpation on each of the samples, using the da Vinci Master Tool Manipulator (MTM) as the master device. The physical palpation of the tissues was done with the da Vinci Patient Side Manipulator (PSM) equipped with an OptoForce 3 DoF sensor, as shown in Fig. 1. The applied force was fed back to the operator through the MTM, serving as a haptic device. After the teleoperated palpation, participants were asked to carry out similar maneuvers using the da Vinci MTM, palpating the virtual models of the selected tissue samples. The same palpation procedure was implemented on the Novint Falcon interface for only the second phase of the trials. The Novint Falcon tests were omitted from Phase I due to the unanimous results of the test using the da Vinci MTM. Participants were allowed to compare the sense of touch during teleoperation and virtual palpation using any of the two devices at any time, and were asked to pair up the real tissues with the virtual ones. The usability study was validated by evaluating the correct answers and comparing them in each of the two cases of the used haptic devices (Novint Falcon and da Vinci MTM).

\section{B. Data Collection and Analysis}

Data collection was done by recording the reaction force of the palpated tissues during their controlled deformation. The thickness of the investigated artificial tissue samples was uniform, $20 \mathrm{~mm}$, the ex-vivo tissue samples had a deviation of $\pm 5 \mathrm{~mm}$ from that dimension. Palpation test on the exvivo tissues were carried out at different points of the surface, indicating that this deviation does not have any significant effect on the measured reaction force data. The indentation depth of all measurements was $4 \mathrm{~mm}$, and regardless of the

\footnotetext{
${ }^{1}$ https://github.com/jhu-dvrk/sawIntuitiveResearchKit/wiki
} 


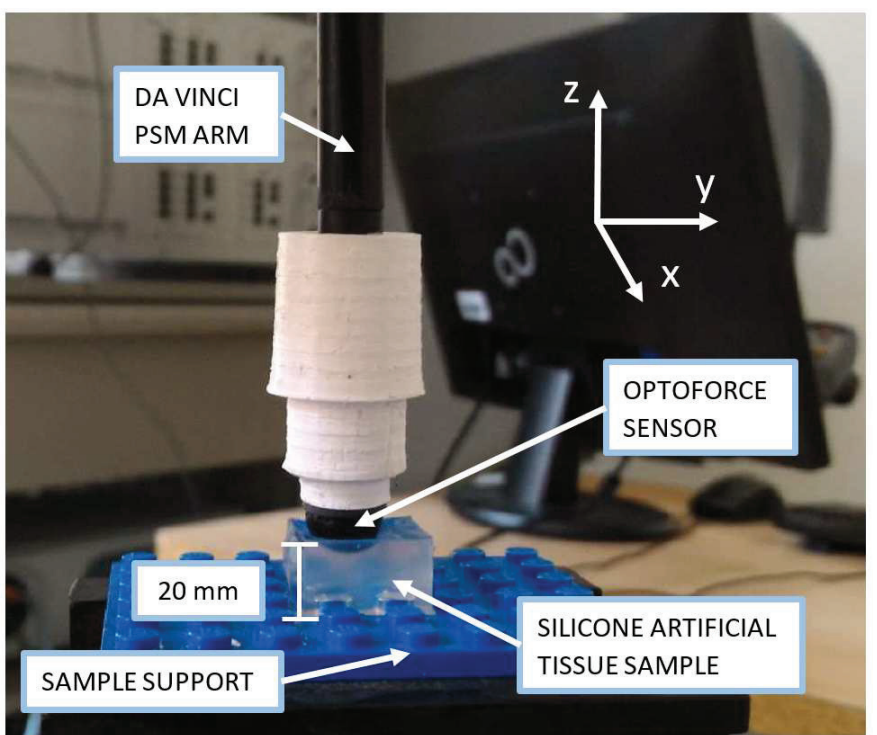

Fig. 1. Indentation tests on a silicone artificial tisse sample, using the da Vinci PSM arm with an OptoForce 3 DoF force sensor.

non-uniform surface deformation, each of the samples were modeled single nonlinear Wiechert-body. These assumptions are valid within the investigated range of deformation, and the tissue parameter values can be generalized to specific stiffness and damping parameters, using the method described in [16]. Each of the samples were subjected to a step-like deformation, where the compression rate was $50[\mathrm{~mm} / \mathrm{s}]$, and the relaxation response was measured for 30 [s]. After the relaxation tests, the samples were compressed at a constant compression rate of $0.5[\mathrm{~mm} / \mathrm{s}]$, and the force response during the compression was recorded. As each sample was tested 5 times for both types deformation tests, the results were averaged and processed for tissue parameter acquisition, as described in [19]. Data collection was done at the sampling rate of $50 \mathrm{~Hz}$, taking into consideration that the da Vinci Research Kit supports $60 \mathrm{~Hz}$ at maximum, while the Novint Falcon and OptoForce devices can easily handle $200-300 \mathrm{~Hz}$ sampling rate as well.

\section{Results}

\section{A. Results for Phase I: User Matches}

1) Data Collection: During Phase I, 3 silicone artificial tissue samples were molded using silorub ds ${ }^{\circledR}$ f-TG silicone, and were softened using Rubosil ${ }^{\text {TM }}$ methyl-silicone oil. Samples $\mathrm{A}, \mathrm{B}$ and $\mathrm{C}$ contained $0 \%, 15 \%$ and $30 \%$ silicone oil, respectively. The ex-vivo chicken breast sample was marked as specimen $\mathrm{D}$, while the ex-vivo chicken liver sample was marked as specimen E. The ex-vivo samples were covered with fresh-keeping film in order to keep the silicone surface of the OptoForce sensor intact. Typical force relaxation response curves and the results of constant compression rate indentation are shown in Fig. 2 and Fig. 3, respectively.

As it is shown in the figures, the stiffness characteristics of the artificial tissue samples are close to linear and there the relaxation phenomenon is negligible. However, Fig. 2 shows

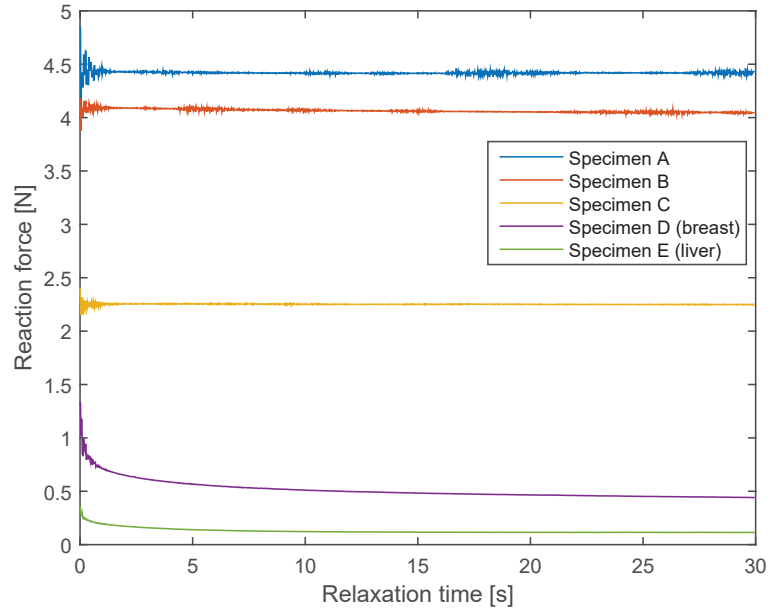

Fig. 2. Typical relaxation force response curves for the specimens used during Phase I, assuming step-like deformation and $4 \mathrm{~mm}$ indentation depth.

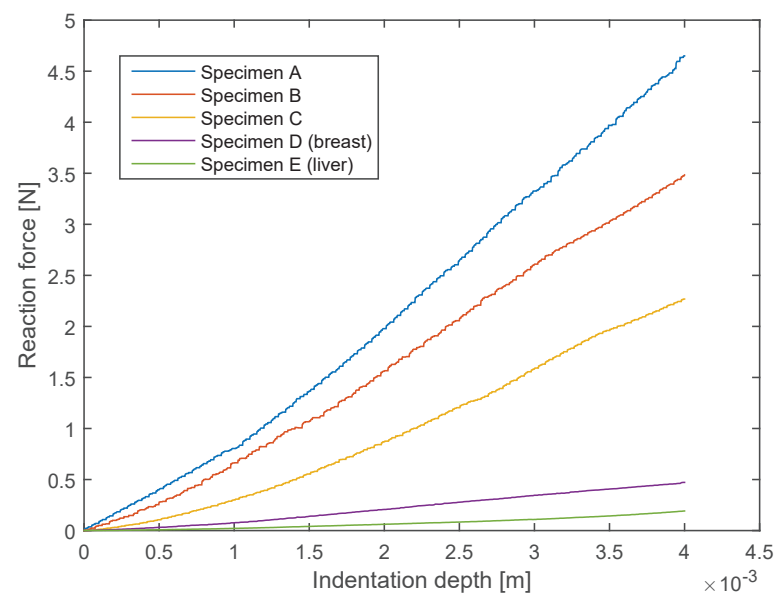

Fig. 3. Typical force response curves for the specimens used during Phase I, assuming constant compression rate deformation and $4 \mathrm{~mm}$ indentation depth.

that the breast and liver samples have a significant decrease in the reaction force due to tissue relaxation, as expected. Tissue parameters were acquired by curve fitting, using the MATLAB (MathWorks Co.) fminsearch function, taking the sum of the root mean square error (RMSE) values from both experimental data sets as the cost function for each sample. The simulated response was calculated by solving the system equations for the iterated parameter values for the nonlinear Wiechert model [17]. The estimated parameters for each of the specimens, based on the results of curve fitting, are shown in Table I. It is important to note that these values are valid for this specific experimental setup, as this paper focuses on the empirical comparison methodology of the investigated samples instead of proposing global parameters value sets for the chosen materials. 
TABLE I

PARAMETER ESTIMATION RESULTS FROM FORCE RELAXATION AND CONSTANT COMPRESSION RATE TESTS DURING PHASE I.

\begin{tabular}{|c|c|c|c|c|c|c|c|c|}
\hline Specimen & $\begin{array}{c}K_{0} \\
{[N / m]}\end{array}$ & $\begin{array}{c}K_{1} \\
{[N / m]}\end{array}$ & $\begin{array}{c}K_{2} \\
{[N / m]}\end{array}$ & $\begin{array}{c}b_{1} \\
{[N s / m]}\end{array}$ & $\begin{array}{c}b_{2} \\
{[N s / m]}\end{array}$ & $\begin{array}{c}\kappa_{0} \\
{\left[m^{-1}\right]}\end{array}$ & $\begin{array}{c}\kappa_{1} \\
{\left[m^{-1}\right]}\end{array}$ & $\begin{array}{c}\kappa_{2} \\
{\left[m^{-1}\right]}\end{array}$ \\
\hline $\mathrm{A}$ & 1093.1 & 1.0616 & 251.09 & 9209.2 & 190 & 0.0056899 & 531.99 & 0.00093 \\
\hline $\mathrm{B}$ & 1002.3 & 1.0861 & 190.38 & 104350 & 145.76 & $3.3147 \mathrm{e}-05$ & 22.679 & $3.3201 \mathrm{e}-05$ \\
\hline $\mathrm{C}$ & 473.13 & 17.062 & 70.787 & 88365 & 66.985 & 58.497 & 234.68 & $7.2614 \mathrm{e}-05$ \\
\hline $\mathrm{D}$ & 1.0001 & 1.0091 & 28.361 & 28.287 & 8.2608 & 577.97 & 969.71 & 10.898 \\
\hline $\mathrm{E}$ & 1.007 & 86.917 & 184.27 & 5375.5 & 4.4498 & 8830.2 & 291.66 & 40.536 \\
\hline
\end{tabular}

2) Tissue Characterization Trials: The virtual Tensor Product (TP) model of each of the specimens was created and implemented into the experimental software background. The da Vinci MTM served as a haptic device, requesting force commands from either directly from the OptoForce sensor or from the virtual model (simulation). The current position and velocity of the MTM were implemented as the input of the system. A force upscaling factor of 10 was applied for helping the participants distinguishing between the models, while the upscaling of the $4 \mathrm{~mm}$ indentation was determined by the participants, restricted by the workspace of the da Vinci MTM (typically a scaling factor of 20-50).

10 subjects participated in the Phase I trials: 8 male and 2 female participants. 1 participant had hands-on surgical experience, 4 came from medical engineering background, while 1 participant had no experience in engineering practice. Participants were aged between 21-40 years, with a median age of 25. At the beginning of each trial, the participants were asked to investigate the virtual models by compressing and releasing them, assessing the tissue properties (stiffness, relaxation, elastic behavior) verbally. Then, after getting familiar with the virtual models, the simulation was switched to the real-time palpation of the tissues. The participants could switch back from the actual palpation to the palpation of the virtual models at any time, and were asked to draw a conclusion, which virtual model (A-E) corresponds to the ex-vivo tissue. For the palpation tests, gravity compensation of the da Vinci MTM was switched off and the orientation of the last 4 axes (tooltip orientation) was locked, allowing only z-axis motion.

Altogether, 20 trials (10 participants for 2 tissue models) were carried out. $95 \%$ of the participants accurately paired the ex-vivo tissue to its corresponding virtual model. One participant mistook specimen D (the chicken breast sample) for the virtual model E (liver sample), the rest of the answers were correct from all participants. Besides the correctness of the answer, some general conclusions were recorded from the participants, listed below:

- Models A and B were significantly stiffer than the rest of the virtual models and the ex-vivo palpated samples: $85 \%$

- The ex-vivo samples had progressive stiffness characteristics, which disclosed models A, B and C from the comparison: $65 \%$

- The reaction force from specimen and model E was very difficult to feel, even in the case of rapid compres- sion: $70 \%$

- Participants spent most of the palpation time differentiating between models $\mathrm{C}$ and $\mathrm{D}$ before drawing the final conclusion, when palpating specimen D: $75 \%$

\section{B. Results for Phase II: User Matches}

Based on the results of the trials of Phase I, Phase II was planned, taking into account the following:

- The liver tissue sample was removed from the investigation due to its low stiffness compared to the silicone samples.

- Specimens A and B were also removed due to their significantly larger stiffness compared to the chicken breast sample.

- Specimen C was kept as a reference and further silicone samples were created by adding more silicone oil during the preparation, until reaching physical limits (saturation of oil in the silicone).

1) Data Collection: During Phase II, 14 silicone artificial tissue samples were created, utilizing the same method as in Phase I. The samples were molded from Silorub ds ${ }^{\circledR} f-$ TG silicone, softening was carried out with a combination of Rubosil $^{\mathrm{TM}}$ methyl-silicone oil and Rubosil ${ }^{\mathrm{TM}}$ silicone grease. Binding was enhanced by using Silorub ds ${ }^{\circledR} \mathrm{K}$ RTV-2 catalyst, adding $2[\mathrm{ml}]$ to every $20[\mathrm{ml}]$ of silicone used. Samples were numbered from 1-14, created with a uniform cubic shape with the edge length of $20 \mathrm{~mm}$. Baking soda was added to sample 13 to further soften the silicone by creating artificial inclusions, and vinegar was added to sample 14 . The volume ratio of the silicone, oil and grease for each of the samples is listed in Table II. The ex-vivo chicken breast sample was marked as specimen 15. All samples were covered with fresh-keeping film in order to keep the silicone oil from damaging the silicone surface of the OptoForce sensor. Typical force relaxation response curves and the results of constant compression rate indentation are shown in Fig. 4 and Fig. 5, respectively. The average force response curves used for model identification are also displayed on the Figures.

2) Tissue Characterization Trials: Before the tissue characterization trials, 6 silicone specimens were selected based on the different behavior of the created tissue samples during the data collection phase. The samples were selected from a wide range of stiffness and maximum reaction force values, taking into account that some of these samples had very 
TABLE II

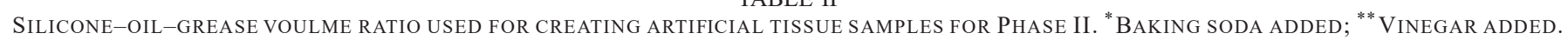

\begin{tabular}{|c|c|c|c|c|c|c|c|c|c|}
\hline Specimen & 1 & 2 & 3 & 4 & 5 & 6 & 7 & 8 & 9 \\
\hline silicone : oil & $1: 3.15$ & $1: 0.5$ & $1: 0.75$ & $1: 1$ & $1: 1.25$ & $1: 1.5$ & $1: 1.85$ & $1: 2.25$ & $1: 2.70$ \\
\hline Specimen & 10 & 11 & \multicolumn{2}{|c|}{12} & \multicolumn{2}{|c|}{13} & 14 \\
\hline silicone : oil : grease & $1: 3.15: 0.5$ & $1: 3.15: 1$ & $1: 3.15: 1.7$ & $1: 3.15: 1.7: 0.8^{*}$ & $1: 3.15: 1.7: 0.8: 0.8^{* *}$ \\
\hline
\end{tabular}
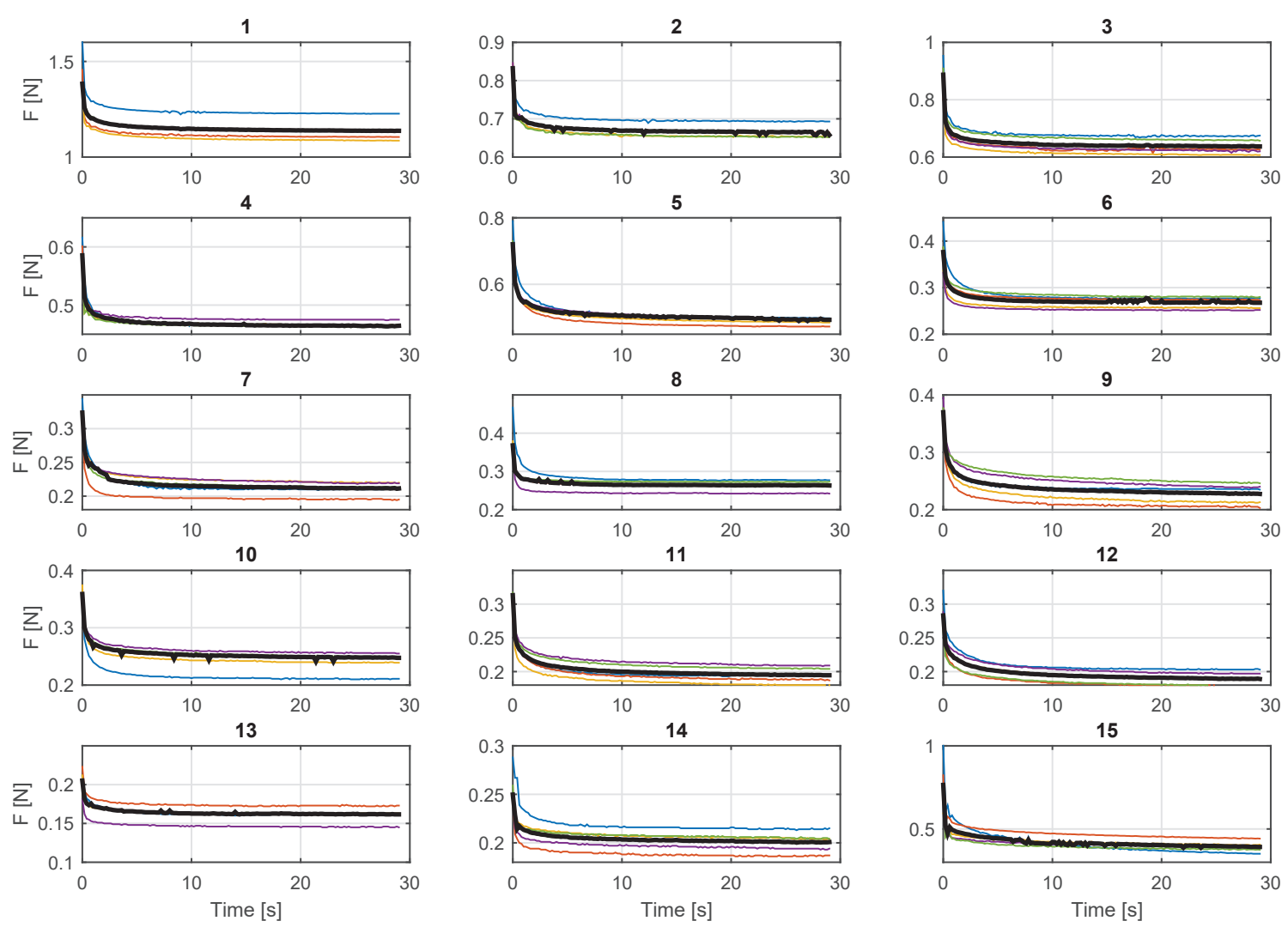

Fig. 4. Measured and average (black) force response curves for the specimens used during Phase II, assuming step-like deformation and 4 mm indentation depth.

similar behavior during relaxation and constant compression rate indentation tests. The virtual TP model of each of the selected samples and the ex-vivo chicken breast sample were created similar to that of Phase I and was implemented into the software. The parameter estimation results for the selected samples from the indentation tests for Phase II are shown in Table III. In order to improve haptic sensation and enhance comparability between the virtual and real specimens, the da Vinci MTML served as a haptic teleoperation device, requesting force commands directly from the Optoforce sensor, while the da Vinci MTMR reflected force values from the virtual model (simulation). The current position and velocity of the MTML and MTML were implemented as the inputs of the real and virtual systems, respectively. A force upscaling factor of 20 was applied for helping the participants distinguishing between the models, upscaling of the $4 \mathrm{~mm}$ indentation was determined by the participants, restricted by the workspace of the da Vinci MTMs (typically a scaling facor of 20-50). The participants were requested to aim for identical ranges for both MTMs, in order to make it easier to compare samples. This way, simultaneously and identically moving the two MTMs, the real and virtual tools reached the tissue surface at the same Z-coordinate of the MTMs.

In Phase II, 23 participants went through the trials. 19 male and 3 female participants. 3 participants had hands-on surgical experience, 15 came from engineering or medical engineering background, 5 of them came from other fields. The participants were aged between 21-60 years, with a median age of 23 years. At the beginning of each trials, the participants were asked to practice individually on both MTM arms in order to 
1
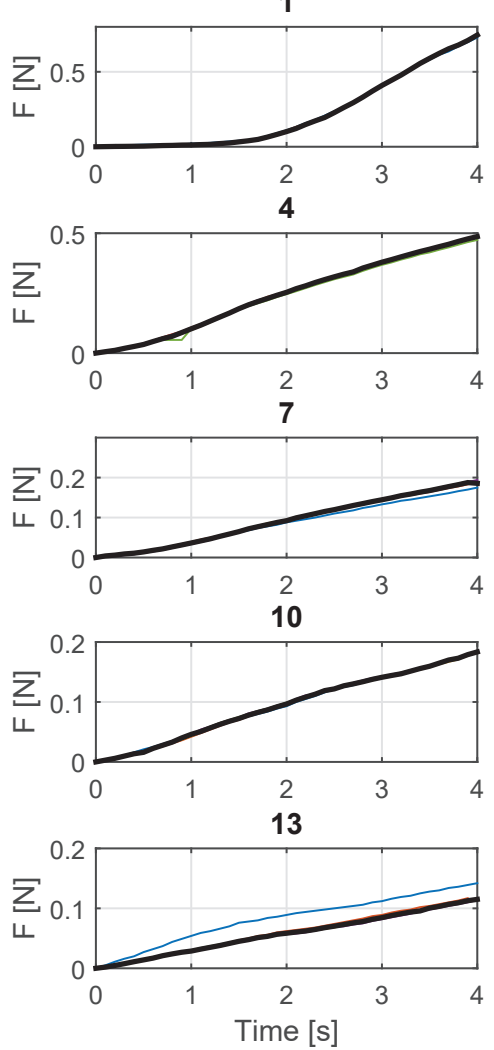

2
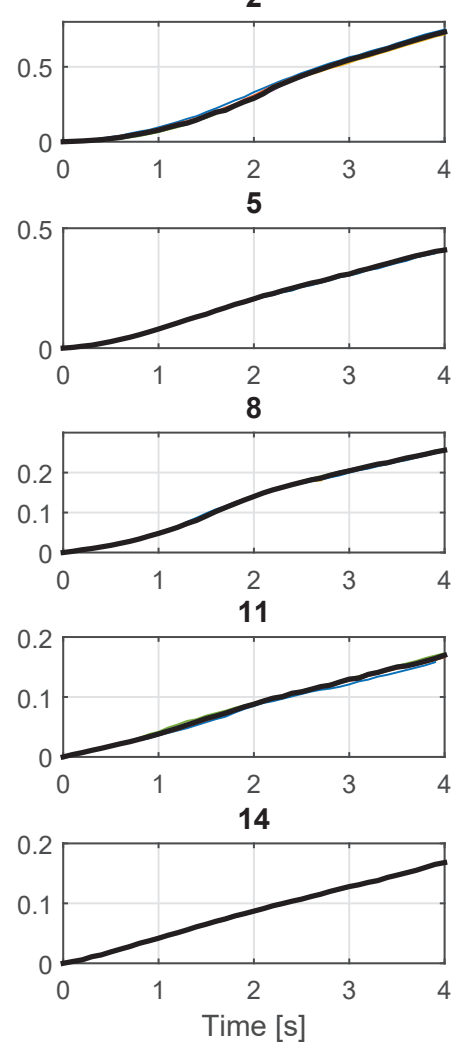

3
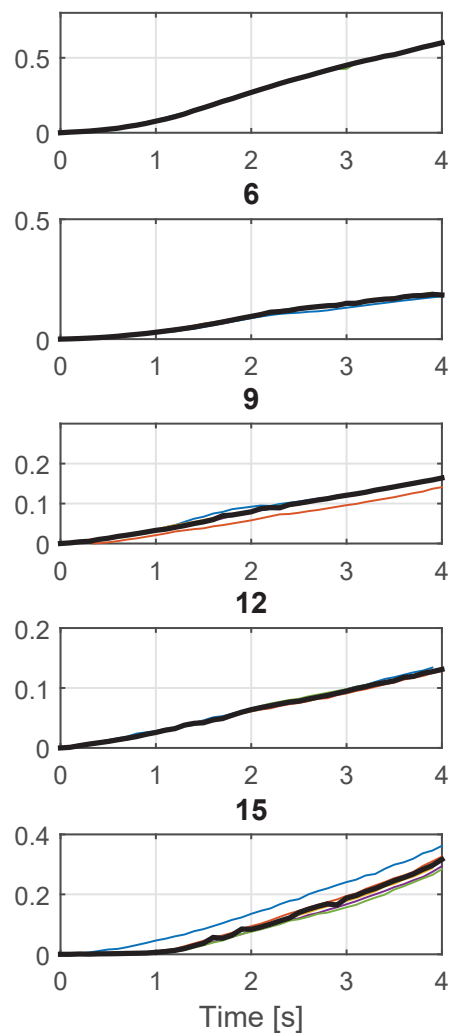

Fig. 5. Measured and average (black) force response curves in Phase II, assuming constant compression rate deformation and 4 mm indentation depth.

TABLE III

PARAMETER ESTIMATION RESUlTS FROM FORCE RELAXATION AND CONSTANT COMPRESSION RATE TESTS DURING PHASE II.

\begin{tabular}{|c|c|c|c|c|c|c|c|c|}
\hline Specimen & $\begin{array}{c}K_{0} \\
{[N / m]}\end{array}$ & $\begin{array}{c}K_{1} \\
{[N / m]}\end{array}$ & $\begin{array}{c}K_{2} \\
{[N / m]}\end{array}$ & $\begin{array}{c}b_{1} \\
{[N s / m]}\end{array}$ & $\begin{array}{c}b_{2} \\
{[N s / m]}\end{array}$ & $\begin{array}{c}\kappa_{0} \\
{\left[m^{-1}\right]}\end{array}$ & $\begin{array}{c}\kappa_{1} \\
{\left[m^{-1}\right]}\end{array}$ & $\begin{array}{c}\kappa_{2} \\
{\left[m^{-1}\right]}\end{array}$ \\
\hline 2 & 115.97 & 2.45 & $5.10 \mathrm{e}-7$ & 238.59 & 13.58 & 90.32 & 747.11 & 19.98 \\
\hline 3 & 99.572 & 0.11764 & 0.101 & 10.001 & 115.64 & 118.85 & $1.56 \mathrm{e} 3$ & 115.51 \\
\hline 5 & 69.62 & 5.58 & 0.10 & 1.07 & 166.37 & 142.92 & 577.81 & 332.75 \\
\hline 8 & 63.95 & 0.48 & $3.44 \mathrm{e}-5$ & 0.61 & 9.07 & 9.94 & 990.62 & 59.83 \\
\hline 10 & 25.54 & $1.00 \mathrm{e}-3$ & $1.00 \mathrm{e}-3$ & 0.63 & 141.44 & 222.95 & $2.31 \mathrm{e} 3$ & 1.55 \\
\hline 12 & 18.79 & 5.65 & 0.17 & 0.29 & 0.07 & 226.61 & 355.52 & 431.72 \\
\hline 15 (ex-vivo $)$ & 18.89 & 0.54 & 13.89 & 329.43 & 17.09 & 217.35 & $1.38 \mathrm{e} 3$ & 16.70 \\
\hline
\end{tabular}

achieve a stable grip, doing so by resting their lower arm on the soft bar da Vinci master console. Once a stable teleoperation was achieved, a randomly chosen virtual model was fed to the MTMR, while the force signal from the OptoForce sensor was constantly fed back to the MTML from the indentation of the ex-vivo chicken breast tissue. On request of the participants, the virtual model was switched to another one of the 7 possibilities (models of the selected 6 artificial tissues and the model of the chicken breast tissue), until they found the best match between the virtual and real tissues according their subjective haptic sensation. Gravity compensation of the da
Vinci MTMs was switched off and the orientation of the last 4 axes were locked, as it was done in Phase I. Fig. 6 summarizes the answers from the participants on which virtual tissue model resembled the most on the behavior of the ex-vivo chicken breast tissue during the palpation tests from the 23 successive trials.

\section{Discussion of the Results}

Results of Phase I indicated that the participants were able to distinguish between the investigated silicone samples and the ex-vivo tissue samples in $95 \%$, which verifies the usability 


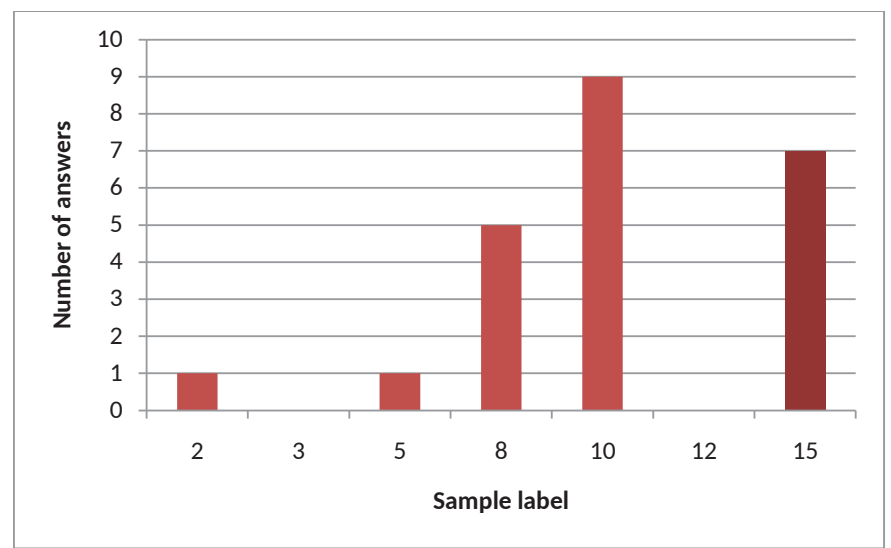

Fig. 6. Tissue characterization results from Phase II, summarizing the participants answers to the question: 'Which virtual tissue model's behavior resembles the most to the ex-vivo chicken breast?'

of the tissue model if there is a significant difference between the mechanical properties of the samples. During Phase II, 7 of 23 participants were able to correctly match the virtual chicken breast model to the ex-vivo tissue, while samples 8 and 10 were chosen 5 and 9 times, respectively. Based on these results, two important conclusions can be drawn:

- The soft tissue model used for representing the tissue behavior is sufficiently good for use in haptic simulators, training and general reaction force estimation. This is based on the observation that a significant percentage $(30 \%)$ of participants were able to match the virtual soft tissue model to the real one.

- Silicon samples 8 and 10 with the proposed composition are capable for modeling soft tissues (in this particular case, chicken breast) in artificially built surgical scenarios or physical phantoms. The conclusion is based on the observation that a large percentage $(60 \%)$ of the participants were unable to distinguish between the physically palpated soft tissue sample and the virtual model of the silicon artificial tissue samples of similar mechanical properties. This similarity is based on the observation of tissue relaxation and constant compression rate tests.

\section{Vi. Conclusion}

Along with force control, the problem of haptic feedback in telesurgical systems remains an open challenge in the related cognitive or ergonomic fields of research. Current surgical teleoperation systems lack haptic feedback capabilities, limiting their usability in everyday practice. This work proposed a validation method for tissue models and their polytopic representation by creating an experimental framework using the da Vinci Research Kit. Furthermore, allowing haptic feedback from the manipulated real tissue, functionality can be extended to surgical simulation using virtual tissue models created by the proposed soft tissue modeling method.

Results of the usability study showed that the proposed nonlinear tissue model very well mimics the mechanical behavior of the ex-vivo tissue both from qualitative and quantitative point of view. This allows one to integrate the model into virtual tissue models used in surgical simulators, where it is critical to have a realistic haptic sensation reflected to the human operator when manipulating the tissues. The study also showed that using a haptic interface, it is challenging to distinguish between artificial silicone tissues and real tissues during teleoperation, indicating that by creating a silicone sample by the methods presented in this work, surgical training can be enhanced by artificial tissue phantoms, though providing realistic haptic sensation to the trainees.

Our future work focuses on the extension of the system database to different ex-vivo tissue models, developing methods for creating artificial silicone samples based on the mechanical properties of these models and on the implementation of this approach into more complex virtual surgical scenarios.

\section{ACKNOWLEDGMENT}

The research was supported by the Ministry of Human Capacities of Hungary. Tamás Haidegger is a Bolyai Fellow of the Hungarian Academy of Sciences. This work has been supported by ACMIT (Austrian Center for Medical Innovation and Technology), which is funded within the scope of the COMET (Competence Centers for Excellent Technologies) program of the Austrian Government. Árpád Takács is a Fellow of the Hungarian National Eötvös Scholarship. The research has been supported by the OTKA PD-114121 Hungarian Scientific Research Fund.

\section{REFERENCES}

[1] G. Tholey, J. P. Desai, and A. E. Castellanos, "Force feedback plays a significant role in minimally invasive surgery: results and analysis," Annals of surgery, vol. 241, no. 1, pp. 102-109, 2005.

[2] M. Ottermo, M. Øvstedal, T. Langø, Ø. Stavdahl, Y. Yavuz, T. Johansen, and R. Mårvik, "The role of tactile feedback in laparoscopic surgery," Surgical Laparoscopy Endoscopy \& Percutaneous Techniques, vol. 16, no. 6, pp. 390-400, 2006

[3] M. Tiwana, S. Redmond, and N. Lovell, "A review of tactile sensing technologies with applications in biomedical engineering," Sensors and Actuators A: physical, vol. 179, pp. 17-31, 2012.

[4] C. E. Reiley, T. Akinbiyi, D. Burschka, D. C. Chang, A. M. Okamura, and D. D. Yuh, "Effects of visual force feedback on robot-assisted surgical task performance," The Journal of thoracic and cardiovascular surgery, vol. 135, no. 1, pp. 196-202, 2008.

[5] A. M. Okamura, L. N. Verner, C. Reiley, and M. Mahvash, "Haptics for robot-assisted minimally invasive surgery," in Robotics research. Springer, 2010, pp. 361-372.

[6] D. Morris, H. Tan, F. Barbagli, T. Chang, and K. Salisbury, "Haptic feedback enhances force skill learning," in Second Joint EuroHaptics Conference and Symposium on Haptic Interfaces for Virtual Environment and Teleoperator Systems (WHC'07). IEEE, 2007, pp. 21-26.

[7] K. Montgomery, C. Bruyns, J. Brown, S. Sorkin, F. Mazzella, G. Thonier, A. Tellier, B. Lerman, and A. Menon, "Spring: A general framework for collaborative, real-time surgical simulation," Studies in health technology and informatics, pp. 296-303, 2002.

[8] H. Delingette, "Toward realistic soft-tissue modeling in medical simulation," Proceedings of the IEEE, vol. 86, no. 3, pp. 512-523, 1998.

[9] M. Tavakoli, A. Aziminejad, R. V. Patel, and M. Moallem, "Highfidelity bilateral teleoperation systems and the effect of multimodal haptics," IEEE Transactions on Systems, Man, and Cybernetics, Part $B$ (Cybernetics), vol. 37, no. 6, pp. 1512-1528, 2007.

[10] Á. Takács, L. Kovács, I. J. Rudas, R.-E. Precup, and T. Haidegger, "Models for force control in telesurgical robot systems," Acta Polytechnica Hungarica, vol. 12, no. 8, pp. 95-114, 2015. 
[11] E. Samur, M. Sedef, C. Basdogan, L. Avtan, and O. Duzgun, "A robotic indenter for minimally invasive measurement and characterization of soft tissue response," Medical Image Analysis, vol. 11, no. 4, pp. 361-373, 2007.

[12] M. Beccani, C. Di Natali, L. J. Sliker, J. A. Schoen, M. E. Rentschler, and P. Valdastri, "Wireless tissue palpation for intraoperative detection of lumps in the soft tissue," IEEE Transactions on Biomedical Engineering, vol. 61 , no. 2, pp. 353-361, 2014.

[13] G. Picinbono, H. Delingette, and N. Ayache, "Nonlinear and anisotropic elastic soft tissue models for medical simulation," in 2001 IEEE International Conference on Robotics and Automation (ICRA), vol. 2. IEEE, 2001, pp. 1370-1375.

[14] A. L. Trejos, J. Jayender, M. Perri, M. D. Naish, R. V. Patel, and R. Malthaner, "Robot-assisted tactile sensing for minimally invasive tumor localization," The International Journal of Robotics Research, 2009.

[15] C. R. Wagner and R. D. Howe, "Force feedback benefit depends on experience in multiple degree of freedom robotic surgery task," IEEE
Transactions on Robotics, vol. 23, no. 6, pp. 1235-1240, 2007.

[16] Á. Takács, I. J. Rudas, and T. Haidegger, "Surface deformation and reaction force estimation of liver tissue based on a novel nonlinear massspring-damper viscoelastic model," Medical \& biological engineering \& computing, vol. 54, no. 10, pp. 1553-1562, 2016.

[17] I. J. Rudas et al., "Nonlinear soft tissue mechanics based on polytopic tensor product modeling," in 2016 IEEE International Symposium on Applied Machine Intelligence and Informatics (SAMI). IEEE, 2016, pp. 211-215.

[18] Á. Takács, S. Jordan, D. Nagy, P. Pausits, T. Haidegger, J. K. Tar, and I. J. Rudas, "Models for force control in telesurgical robot systems," Acta Universitatis Sapientae Electrical and Mechanical Engineering, vol. 14, no. 6, pp. 20-34, 2015.

[19] A. Takacs, P. Galambos, P. Pausits, I. J. Rudas, and T. Haidegger, "Nonlinear soft tissue models and force control for medical cyberphysical systems," in 2015 IEEE International Conference on Systems, Man, and Cybernetics (SMC). IEEE, 2015, pp. 1520-1525. 